\title{
Quinolones to treat acute bacterial exacerbation of chronic bronchitis in TB endemic areas: a word of caution
}

\section{To the Editors:}

The meta-analysis by SIEMPOS et al. [1] has shown equivalent efficacy of macrolides, quinolones and amoxicillin/clavulanate in the treatment of patients with acute bacterial exacerbation of chronic bronchitis (ABECB). Although quinolones are associated with better microbiological success and fewer recurrences of $\mathrm{ABECB}$, certain points merit further discussion, especially in tuberculosis (TB) endemic areas.

Most of the randomised controlled trials included in this metaanalysis were published from the Western world, where TB is not widespread. In areas endemic of TB, Mycobacterium tuberculosis has been isolated from the sputum specimen of $1.5-5 \%$ of cases with acute exacerbations of chronic obstructive pulmonary disease, thus reflecting the high prevalence of active $\mathrm{TB}$ as a precipitating cause for acute exacerbation [2, 3].

Besides providing good coverage for the usual bacterial organisms causing $\mathrm{ABECB}$, quinolones have potent antimycobacterial properties. Their use to treat $A B E C B$ can lead to temporary improvement in symptoms of pulmonary $\mathrm{TB}$, thus delaying the diagnosis and prolonging the patient's infectivity. In a study by Dooley et al. [4], initial empiric therapy with a quinolone was associated with a delay in the initiation of appropriate anti-TB treatment (21 versus 5 days). This delay in the diagnosis of active TB translates not only into greater patient morbidity and mortality, but also in increasing the spread of TB in the community.

Quinolones are essential drugs in the treatment of drugresistant TB. Widespread and indiscriminate use of quinolones, due to their broad-spectrum antibacterial coverage and relatively low cost, is likely to rapidly enhance their resistance to most of the susceptible organisms in the community, including mycobacteria. Reports of quinolone-resistant TB [5] and extensively drug-resistant (XDR)-TB [6] are pouring in.

XDR-TB has now been identified in all regions of the world, but it is most prevalent in Asia and Eastern Europe [5]. A recent study carried out in a tertiary care hospital in India [7] found that XDR-TB accounts for $8 \%$ of multidrug-resistant cases, compared with $\sim 4 \%$ in the USA.

Therefore, in light of the potential of masking active tuberculosis and the threat of an emerging epidemic of extensively drug-resistant tuberculosis, the use of quinolones should be restricted in tuberculosis endemic areas.

\section{A. Singh}

Pulmonary and Critical Care Medicine, Christian Medical College and Hospital, Ludhiana, India.

\section{STATEMENT OF INTEREST}

None declared.

\section{REFERENCES}

1 Siempos II, Dimopoulos G, Korbila IP, Manta K, Falagas ME. Macrolides, quinolones and amoxicillin/clavulanate for chronic bronchitis: a meta-analysis. Eur Respir J 2007; 29: 1127-1137.

2 Mohan A, Premanand R, Reddy LN, et al. Clinical presentation and predictors of outcome in patients with severe acute exacerbation of chronic obstructive pulmonary disease requiring admission to intensive care unit. BMC Pulm Med 2006; 6: 27.

3 Ko FW, Ng TK, Li TS, et al. Sputum bacteriology in patients with acute exacerbations of COPD in Hong Kong. Respir Med 2005; 99: 454-460.

4 Dooley KE, Golub J, Goes FS, Merz WG, Sterling TR. Empiric treatment of community-acquired pneumonia with fluoroquinolones, and delays in the treatment of tuberculosis. Clin Infect Dis 2002; 34: 1607-1612.

5 Sullivan EA, Kreiswirth BN, Palumbo L, et al. Emergence of fluoroquinolone-resistant tuberculosis in New York City. Lancet 1995; 345: 1148-1150.

6 Centers for Disease Control and Prevention (CDC)., Emergence of Mycobacterium tuberculosis with extensive resistance to second-line drugs-worldwide, 2000-2004. MMWR Morb Mortal Wkly Rep 2006; 55: 301-305.

7 Jain S, Rodrigues C, Mehta A, Udwadia ZF. High Prevalence of XDR TB from a tertiary care hospital in India, Session B98, Abstract No. 1398. http:/ / www.thoracic.org/sections/ meetings-and-courses/international-conference/2007/conferenceprogram/conference-sessions/detail.cfm?s=B98. Date created: May 21, 2007. Date last accessed: June 11, 2007.

DOI: $10.1183 / 09031936.00070407$ 